\title{
A crowdsourced intervention to promote hepatitis $B$ and $C$ testing among men who have sex with men in China: study protocol for a nationwide online randomized controlled trial
}

Thomas Fitzpatrick ${ }^{1 *}$ (D) Kali Zhou ${ }^{2}$, Yu Cheng ${ }^{3}$, Po-Lin Chan ${ }^{4}$, Fuqiang Cui ${ }^{5}$, Weiming Tang ${ }^{6}$, Katie R Mollan ${ }^{7}$, Wilson Guo and Joseph D Tucker ${ }^{6}$

\begin{abstract}
Background: The World Health Organization recommends all men who have sex with men (MSM) receive Hepatitis B Virus (HBV) and Hepatitis C Virus (HCV) testing. MSM in China are a high-risk group for HBV and HCV infection, but test uptake is low. Crowdsourcing invites a large group to solve a problem and then shares the solution with the public. This nationwide online randomized controlled trial will evaluate the effectiveness of a crowdsourced intervention to increase HBV and HCV testing among MSM in China.

Methods: Seven hundred MSM will be recruited through social media operated by MSM organizations in China. Eligible participants will be born biologically male, age 16 years or older, report previous anal sex with another man, and reside in China. After completing a baseline online survey, participants will be randomly assigned to intervention or control arms with a 1:1 allocation ratio. The intervention will include two components: (1) a multimedia component will deliver two videos and two images promoting HBV and HCV testing developed through a crowdsourcing contest in China; (2) a participatory component will invite men to submit suggestions for how to improve crowdsourced videos and images. The control arm will not view any images or videos and will not be invited to submit suggestions. All participants will be offered reimbursement for HBV and HCV testing costs. The primary outcome is HBV and HCV test uptake confirmed through electronic submission of test report photos within four weeks of enrolment. Secondary outcomes include self-reported HBV and HCV test uptake, HBV vaccination uptake, and change in stigma toward people living with HBV after four weeks. Primary and secondary outcomes will be calculated using intention to treat and as-exposed analyses and compared using two-sided 95\% confidence intervals.

Discussion: Few previous studies have evaluated interventions to increase HBV and HCV testing in middle-income countries with a high burden of hepatitis. Delivering a crowdsourced intervention using social media is a novel approach to increasing hepatitis testing rates. HBV and HCV test uptake will be confirmed through test report photos, avoiding the limitations of self-reported testing outcomes.
\end{abstract}

Trial registration: NCT03482388 (29 March 2018).

Keywords: Hepatitis B virus (HBV), Hepatitis C virus (HCV), Testing, Men who have sex with men (MSM), Crowdsourcing, China

\footnotetext{
* Correspondence: tsfitz@uw.edu

'School of Medicine, University of Washington, Seattle, WA, USA

Full list of author information is available at the end of the article
}

(c) The Author(s). 2018 Open Access This article is distributed under the terms of the Creative Commons Attribution 4.0 International License (http://creativecommons.org/licenses/by/4.0/), which permits unrestricted use, distribution, and reproduction in any medium, provided you give appropriate credit to the original author(s) and the source, provide a link to the Creative Commons license, and indicate if changes were made. The Creative Commons Public Domain Dedication waiver (http://creativecommons.org/publicdomain/zero/1.0/) applies to the data made available in this article, unless otherwise stated. 


\section{Background}

Hepatitis B Virus (HBV) and Hepatitis C Virus (HCV) are substantial contributors to global morbidity and mortality, together accounting for more deaths annually than HIV [1]. Most individuals affected by chronic viral hepatitis are in low- and middle-income countries (LMIC) [2]. China is a middle-income country with a high burden of hepatitis [3]. HBV and HCV prevalence among the general population in China is $7 \%$ and $0.8 \%$, respectively, and China alone has more persons living with chronic viral hepatitis than Europe and the Americas combined [2-4].

Men who have sex with men (MSM) are a group at increased risk for $\mathrm{HBV}$ and $\mathrm{HCV}$ infection [5, 6]. Prevalence of HBV and HCV is higher among MSM than the general population in multiple contexts, [7-10] including China $[11,12]$. As a result, the World Health Organization (WHO) guidelines recommend all MSM receive $\mathrm{HBV}$ and $\mathrm{HCV}$ testing at least once [13]. Testing is the key first step in the viral hepatitis care continuum and is necessary for infected persons to be linked to care, initiated on treatment, and to achieve viral suppression or cure. Expedited linkage to the viral hepatitis care continuum can reduce liver-related deaths, [14, 15] and hepatitis testing can identify those susceptible to $\mathrm{HBV}$ infection and facilitate vaccination [16].

Despite the importance of testing, HBV and HCV testing rates among MSM in China are low. A 2017 nationwide online survey of MSM in China found 59\% had never $\mathrm{HCV}$ tested, and among those with no or uncertain $\mathrm{HBV}$ vaccination, $62 \%$ had never $\mathrm{HBV}$ tested [17]. Chinese MSM who had not HBV or HCV tested were also less likely to have received screening for HIV and other sexually transmitted infections (STI) [17]. Novel methods of increasing hepatitis testing and linkage to HIV/STI testing services among MSM are needed.

$\mathrm{HBV}$ and $\mathrm{HCV}$ testing services can be potentially strengthened using crowdsourcing. Crowdsourcing invites a large group to solve a problem and then shares the solution with the public [18]. This bottom-up approach can spur innovation through collecting and refining a wide-range of creative solutions from a diverse group of contributors [13]. Additionally, crowdsourcing engages communities in the development of responses to important issues, thereby increasing community participation and producing more locally relevant solutions [19, 20].

Crowdsourcing has been used to identify health innovation by many funders, including the United States National Institutes of Health, the English National Health Service, the Bill and Melinda Gates Foundation, and the United States government [21, 22]. Non-inferiority randomized controlled trials (RCTs) have shown that crowdsourcing is a cost-effective tool for increasing HIV testing uptake and is as efficacious as evidence-based health marketing materials [23, 24]. Additionally, social media interventions that involve active participation and content generation, such as crowdsourcing, have been associated with higher rates of HIV and STI testing compared to passive testing campaigns [25].

Few previous studies have evaluated interventions to increase hepatitis testing in LMIC, and none of these have targeted MSM or used a crowdsourcing approach [26]. The purpose of this RCT is to address this gap in the current literature and evaluate the impact of a crowdsourced intervention on $\mathrm{HBV}$ and $\mathrm{HCV}$ test uptake among MSM in China.

\section{Objectives}

Specific aim 1: To compare confirmed HBV and HCV test uptake as a composite endpoint between MSM randomly assigned to a crowdsourced intervention and MSM randomly assigned to control.

Hypothesis 1: MSM assigned to the crowdsourced intervention will have a higher rate of $\mathrm{HBV}$ and $\mathrm{HCV}$ test uptake compared to MSM in the control arm.

Specific aim 2: To compare change in stigma toward people living with HBV between MSM randomly assigned to a crowdsourced intervention and MSM randomly assigned to control.

Hypothesis 2: MSM assigned to the crowdsourced intervention will have greater reduction in stigma toward people living with $\mathrm{HBV}$ compared to MSM in the control arm.

Specific aim 3: To compare self-reported linkage to clinical visits about hepatitis, HBV vaccination, and HIV/STI testing services between MSM randomly assigned to a crowdsourced intervention and MSM randomly assigned to control.

Hypothesis 3: MSM assigned to the crowdsourced intervention will have improved linkage to clinical visits about hepatitis, HBV vaccination, and HIV/STI testing services compared to MSM in the control arm.

\section{Methods}

\section{Trial design and timeline}

This study will be an online superiority RCT. After completing a baseline survey to assess eligibility, enrolled participants will be randomly assigned in a 1:1 ratio into the intervention or control arms. Men randomly assigned to the intervention arm will be asked to view four crowdsourced images and videos promoting hepatitis testing over 8 days. After viewing each image or video men will be invited to submit suggestions for how to better tailor the crowdsourced materials to the MSM community. Men randomly assigned to the control arm will view no materials and will not be invited to submit 
suggestions. Participants can submit photos of hepatitis test reports to confirm HBV and HCV test uptake at any time after enrolment. After 3 weeks men will be sent a message asking them to complete a follow-up survey within 1 week. All participants will be assessed for primary and secondary outcomes at 4 weeks (28 days) post-enrolment (Table 1). The study is anticipated to begin enrolment in April 2018 and finish data collection by June 2018. A flowchart summarizing trial design and timeline is presented in Fig. 1.

\section{Study setting and recruitment}

This study will be conducted online. All recruitment, enrolment, intervention delivery, and data collection will occur through mobile phones and social media smartphone apps. Nearly $70 \%$ of the adult population in China owns a smartphone, and 95\% of all netizens in China access the internet using a mobile phone [27, 28]. In this study, participants will be recruited through an announcement promoted via social media accounts operated by several local MSM organizations. The announcement will include a link connecting men to the online baseline survey to be completed using a smartphone. The link will remain active until 700 eligible men have enrolled.

Once enrolled, WeChat (Tencent Holdings Limited, Shenzhen, China) will be used to deliver messages and collect confirmatory photos of hepatitis test reports from participants. Additionally, men randomly assigned to the intervention arm will view crowdsourced images and videos through WeChat. WeChat is a multipurpose social media smartphone app with messaging and

Table 1 Primary and secondary outcomes with definitions

\begin{tabular}{ll}
\hline Primary outcome & Definition \\
\hline Confirmed HBV and HCV test uptake & Defined as men who had both HBsAg test uptake and anti-HCV IgG test uptake confirmed \\
& through electronic submission of a test report photo showing serology results, age of tester, \\
& sex of tester, and date of test within four weeks of enrolment
\end{tabular}

Secondary outcomes

Confirmed HBV test uptake

Defined as men who had HBsAg test uptake confirmed through electronic submission of a test report photo showing serology results, age of tester, sex of tester, and date of test within four weeks of enrolment

Confirmed HCV test uptake

Defined as men who had anti-HCV IgG test uptake confirmed through electronic submission of a test report photo showing serology results, age of tester, sex of tester, and date of test within four weeks of enrolment

Self-reported HBV test uptake

Defined as men who had HBsAg uptake within four weeks of enrolment, self-reported in follow-up survey

Self-reported HCV test uptake

Defined as men who had anti-HCV IgG uptake within four weeks of enrolment, self-reported in follow-up survey

HBV vaccination uptake

Defined as men who had receipt of at least a one dose of the HBV vaccine within four weeks of enrolment, self-reported in follow-up survey

HBV vaccination uptake among men with confirmed susceptibility to HBV infection

Defined as men with negative HBsAg and negative anti-HBs results confirmed through elec tronic submission of a test report photo showing serology results, who had receipt of at least one dose of the HBV vaccine within four weeks of enrolment, self-reported in follow-up survey

HIV test uptake

Chlamydia test uptake

Gonorrhoea test uptake

Syphilis test uptake

Change in stigma toward people living with HBV

Visit with a physician after hepatitis test uptake
Defined as men who had HIV test uptake within four weeks of enrolment, self-reported in follow-up survey

Defined as men who had chlamydia test uptake within four weeks of enrolment, self-reported in follow-up survey

Defined as men who had gonorrhoea test uptake within four weeks of enrolment, selfreported in follow-up survey

Defined as men who had syphilis test uptake within four weeks of enrolment, self-reported in follow-up survey

Continuous variable, defined as difference between Toronto Chinese HBV Stigma Scale score assessed at follow-up and baseline. Stigma toward people living with HBV will be measured at baseline and follow-up using 20 survey items that are each on a five point Likert scale. The 20 items were originally developed as the Toronto Chinese HBV Stigma Scale (potential range of 20-100), which has been previously validated and correlated to HBV testing behaviours among Chinese populations [39]. Decreased stigma toward people living with HBV will be defined as a mean composite score that is less at follow-up compared to baseline.

Defined as men who had HBV and/or HCV test uptake and saw a physician to discuss hepatitis test results within four weeks of enrolment, self-reported in follow-up survey 


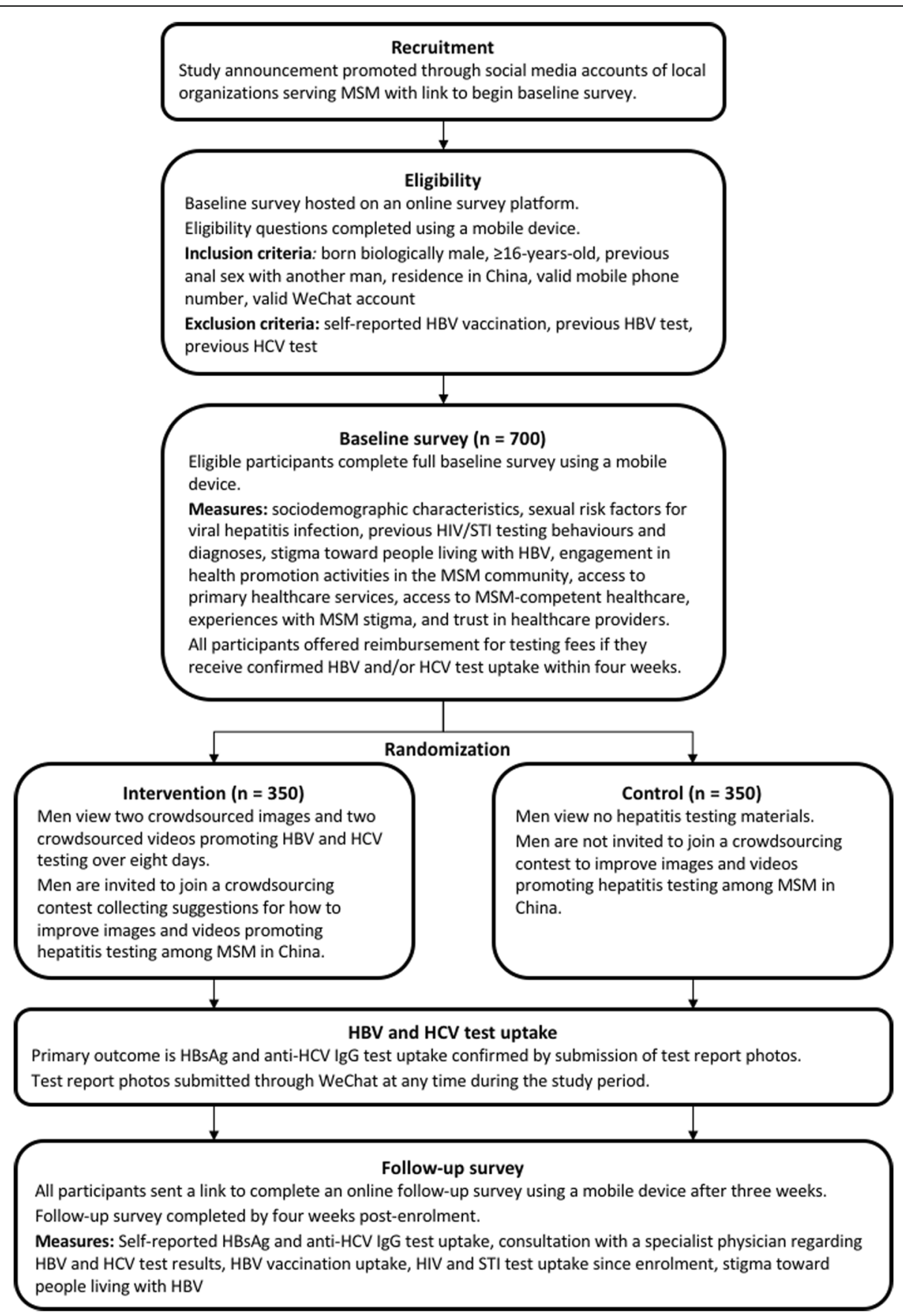

Fig. 1 Trial design to evaluate a crowdsourced intervention to increase hepatitis testing among MSM in China

multimedia functionality. The app had approximately 900 million daily active users in 2017, most of whom live in China, and is commonly used to send and share private messages, social media posts, images, and videos [29].

\section{Eligibility criteria}

Potential participants must first agree to an online informed consent before beginning the baseline online survey. Inclusion criteria are being born biologically male, age 16 years or older, self-reported previous anal sex with another man, and current residence in China. Men who report past $\mathrm{HBV}$ vaccination, $\mathrm{HBV}$ testing, or $\mathrm{HCV}$ testing will be excluded. Those who report uncertain past $\mathrm{HBV}$ vaccination, $\mathrm{HBV}$ testing, or $\mathrm{HCV}$ testing will be considered eligible. All eligible participants must provide a working unique mobile phone number and WeChat account to be enrolled. 


\section{Randomization and allocation}

Once enrolled, participants will be assigned in a 1:1 ratio to intervention or control through a randomization procedure using permuted blocks. SAS software (Cary, North Carolina, USA) will be used to create the allocation sequence using the PROC PLAN and RANUNI functions. The allocation sequence will be applied to participants sequentially in the order in which they were enrolled. This RCT is not blinded. Participants will be able to determine their assignment based on whether they received intervention materials during the study period. The primary investigator (TF) will be aware of randomization assignment because intervention materials will be delivered through WeChat.

\section{Intervention and control}

Men randomly assigned to the intervention arm will receive a two-part crowdsourced intervention. The multimedia component of the intervention will deliver two images and two one-minute videos to men through WeChat. One image or video will be delivered every other day after enrolment, for a total of 8 days. Intervention images and videos were developed through a public nationwide crowdsourcing contest conducted in China in 2017.

A steering committee consisting of representatives from 13 national and international organizations was formed to organize a contest to solicit multimedia materials that promote $\mathrm{HBV}$ and $\mathrm{HCV}$ testing and reduce stigma toward people living with viral hepatitis. An initial public call for images and one-minute videos was promoted through social media and partner organizations. In total, 168 submissions were collected between February and May 2017. All submissions were first evaluated for eligibility and were then reviewed by a judging panel composed of steering committee members. Judges evaluated submissions on a 10-point scale based on each submission's innovation and creativity, capacity to promote hepatitis testing, and potential to increase social media responsiveness. The twelve highest scoring entries were selected as semi-finalists and provided with feedback for improvement. Semi-finalists then had the opportunity to resubmit their images or videos. Eight submissions (four images and four videos) were selected as contest finalists, with five receiving third place, two receiving second place, and one receiving first place.

To select the finalist entries most appropriate for MSM in China, an online poll was conducted in August 2017. The poll was promoted through local MSM organizations and their social media accounts. Thirty MSM were asked to rank the four finalist videos, and a separate $30 \mathrm{MSM}$ were asked to rank the four finalist images. The two top-ranked images and videos from this poll were selected as materials to be used in the intervention arm of the RCT (intervention images are available in Supplementary Materials as Additional file 1 and Additional file 2).

Because the strength of crowdsourcing includes not only the inflow of creative solutions and new ideas, but also facilitation of active participation and community engagement, the intervention will also include a participatory component. After viewing each image and video, men will be invited to participate in a crowdsourcing contest. Men can submit 50- to 200-word suggestions for how to improve the intervention images and videos to convince more MSM in China to receive hepatitis testing. A contest steering committee composed of hepatitis experts and community representatives will judge each submission on a 10-point scale, and the highest-scoring eight submissions will receive prizes.

Men randomly assigned to control will not view any images or videos and will not be invited to submit ideas for improving hepatitis testing promotional materials. All participants will be informed at enrolment that costs of HBV and HCV testing during the four-week study period can be reimbursed. Because this study is designed as a pragmatic trial with results that will be generalizable to multiple settings, men will be allowed to receive hepatitis testing at any hospital, clinic, or healthcare centre of their choosing.

\section{Outcomes}

HBsAg and anti-HCV IgG test uptake confirmed by test report photo at 4 weeks post-enrolment is the primary outcome of this study. This dichotomous outcome has two component endpoints (HBsAg test uptake and anti-HCV IgG test uptake) that constitute a single composite endpoint. Selection of HBsAg and anti-HCV IgG as serological assays is based on WHO testing guidelines [13]. A participant will only be counted as having the primary outcome if he has submitted a photo to researchers through WeChat or the online follow-up survey that clearly displays tester age, tester sex, test date, and HBsAg and anti-HCV IgG results. If submitted HBV test report photos also include anti-HBs and anti-HBc serological assays, these results will be recorded. Test date must fall within the four-week period (28 days) after enrolment for test uptake to be confirmed.

Confirmed HBsAg and anti-HCV IgG test uptake as independent component endpoints are secondary outcomes. Secondary outcomes also include the following self-reported items on the follow-up survey: HBsAg test uptake, anti-HCV IgG test uptake, HIV test uptake, chlamydia test uptake, gonorrhoea test uptake, syphilis test uptake, visit with specialist physician after HBV or $\mathrm{HCV}$ testing, and change in stigma toward people living with HBV during the four-week study period. 
Self-reported HBV vaccination uptake among all participants and those with confirmed susceptibility to HBV infection (negative anti-HBs and negative HBsAg by test report photo) are also secondary outcomes. Definitions for all study outcomes are provided in Table 1.

\section{Sample size}

A pilot study was conducted in November 2017 to inform sample size calculations. Pilot results are detailed in Supplementary Materials (see Additional file 3). Assuming a confirmed $\mathrm{HBV}$ and $\mathrm{HCV}$ testing response of $8.5 \%$ among intervention participants, $3.0 \%$ among control participants, a two-sided significance level of 5\%, and $10 \%$ not evaluable (e.g., missing data), a total sample size of 674 men would be needed to have $80 \%$ power for an exact test. Power was calculated in SAS version 14.2 using the twosamplefreq statement in PROC POWER. The total sample size was rounded up to 700 for convenience, which corresponds to approximately $82 \%$ power under the above assumptions.

\section{Data collection and measures}

Baseline and follow-up surveys will be delivered to mobile devices and completed online using Wenjuanxing (Sojump, Shanghai, China), a web-based survey tool that meets industry standards for security and functionality. Both surveys were written in Chinese, and 12 local MSM were asked to provide feedback to ensure questions are clear and culturally appropriate.

The baseline survey will first ask respondents to answer questions regarding eligibility criteria. If eligible, respondents will be asked to complete the remainder of the survey. The baseline survey will include the following domains: sociodemographic characteristics, sexual risk factors for viral hepatitis infection, previous HIV/STI testing behaviours and diagnoses, stigma toward people living with HBV, engagement in health promotion activities in the MSM community, access to primary healthcare services, access to MSM-competent healthcare, experiences with MSM stigma in healthcare settings, and trust in healthcare providers. Twenty-eight questions will measure sexual risk factors for viral hepatitis infection, which were adapted from the validated HCV-MOSAIC risk score for HCV infection among MSM [30]. Stigma toward people living with HBV will be assessed using the Toronto Chinese HBV Stigma Scale, a 20-item index validated for Chinese-speaking populations [12]. Engagement in health promotion activities will be measured through six items used in previous evaluations of crowdsourced interventions [31]. Access to MSM-competent healthcare will be assessed through three questions adapted from a recent survey investigating MSM-competent physicians in China [32]. Seven questions adapted from research in the United States will be used to measure experiences with MSM stigma in healthcare settings and trust in healthcare providers [33, 34].

Participants will receive a WeChat message 3 weeks after enrolment asking them to complete a follow-up survey within 1 week. The follow-up survey will collect information regarding the following self-reported outcomes: HBsAg and anti-HCV IgG test uptake, consultation with a specialist physician regarding $\mathrm{HBV}$ and $\mathrm{HCV}$ test results, HBV vaccination uptake, and HIV and STI test uptake since enrolment. Stigma toward people living with HBV will be reassessed. Participants will be asked to report their exposure to intervention materials and behaviours sharing intervention materials on social media during the four-week study period. Men in the intervention and control arms will receive the same follow-up survey. Men who have not completed the follow-up survey 2 days prior to the end of the four-week study period will be sent a reminder message. Both the baseline and follow-up surveys are expected to take less than $10 \mathrm{~min}$ to complete and are available in Supplementary Materials (see Additional file 4 and Additional file 5).

At any time after enrolment and throughout the four-week study period participants can submit photos of their HBV and HCV test reports through WeChat. Test report photos can also be uploaded along with the follow-up survey. The following information will be extracted and recorded from submitted photos: tester age, tester sex, date of test, and test result. No other personally identifiable information will be extracted. Mobile phone numbers and WeChat accounts will be used to link information extracted from test report photos to baseline and follow-up survey responses.

Among men assigned to the intervention arm, suggestions for improving crowdsourced images and videos will be collected through a web-based survey form hosted on Wenjuanxing. Information on contest participation will be linked to baseline and follow-up survey responses using mobile phone numbers and WeChat accounts.

\section{Confidentiality}

Survey data will be collected through and stored on a secure online survey platform (Wenjuanxing). Photos of test reports will be collected through a secure online messaging platform (WeChat) or a secure online survey platform (Wenjuanxing). Participants will be instructed to cover or obscure their name and other personal identifying information on the test report to protect their privacy. Data will be transmitted using 128-bit encryption across the internet and mobile data networks. Responses to both baseline and follow-up surveys, including participant mobile phone numbers and WeChat 
account numbers, will be stored on a secure server which can be accessed with login information known only to the research team.

\section{Incentives and reimbursement}

Participants who complete the initial survey will receive 4.73 USD (30 RMB), and those who complete the follow-up survey will receive an additional 7.89 USD (50 RMB). All testing costs up to 11.04 USD (70 RMB) for HBV and 6.31 USD (40 RMB) for HCV will be reimbursed to men who submit photos of their test results. Reimbursement amounts are based on prices of national standard HBV testing items (qualitative $\mathrm{HBsAg}$, Anti-HBs, and Anti-HBc serological assay) and HCV testing items (qualitative anti-HCV IgG serological assay), respectively.

Men in the intervention arm who submit suggestions for improving hepatitis testing promotion materials will be eligible to receive contest prizes. Prizes of 78.88 USD (500 RMB), 31.55 USD (200 RMB), and 15.77 USD (100 $\mathrm{RMB}$ ) will be distributed to one first-place finalist, two second-place finalists, and five third-place finalists, respectively. All incentives and reimbursements will be distributed as electronic money transfers through WeChat.

\section{Monitoring}

A data monitoring committee will not be formed for this RCT because potential for harm to participants is minimal. If any person feels they have experienced an adverse event or unwanted effect from participating in this RCT, they can withdraw at any time. A telephone number and WeChat account will be provided to participants to contact the primary investigator with questions or concerns.

\section{Data analysis}

Primary analysis

Participant baseline characteristics and outcomes will be summarized using descriptive statistics. The primary analysis will evaluate the hypothesis that the crowdsourced intervention is superior to control (i.e. no exposure) in increasing $\mathrm{HBV}$ and $\mathrm{HCV}$ testing among MSM in China. The proportion of men with confirmed HBsAg and anti-HCV IgG test uptake will be calculated for the intervention and control arms separately using a two-sided binomial 95\% CI. The difference in proportions with test uptake will be calculated (intervention control) with a corresponding Wald 95\% CI. A Z-test will also be performed to compare the difference in proportions with test uptake between the intervention and control arms. If the 95\% CI for the difference in probabilities is entirely above zero, then the intervention will be declared superior to control. If testing proportions are lower than anticipated such that statistical assumptions of the Wald CI are not met, then an exact 95\% CI for the difference in probabilities will be used.

The effect of the intervention on HBV and HCV testing will first be evaluated using an intention to treat analysis that includes men lost to follow-up. Those who are lost to follow-up or do not respond will be counted as having not tested for $\mathrm{HBV}$ and $\mathrm{HCV}$. An as-exposed analysis will also be performed that reassigns participants based on their self-reported exposure to the intervention materials and adjusts for potential confounders measured in the baseline survey.

\section{Effect measure modification analysis}

Effect measure modification analyses will evaluate whether the effect of the intervention on confirmed $\mathrm{HBV}$ and $\mathrm{HCV}$ testing varied in relation to the following factors measured at baseline: (1) engagement in health promotion activities in the MSM community, (2) access to primary healthcare services, (3) access to MSM-competent healthcare, (4) experiences with MSM stigma in healthcare settings, (5) trust in healthcare providers, and (6) age cohort (16-24 years old, 25-29 years old, and $\geq 30$ years old). Age cohorts were selected based on previous epidemiological studies investigating $\mathrm{HBV}$ infection and vaccine-associated immunity among MSM in China [12].

\section{Missing data plan}

Any participants lost to follow-up will be included and assumed to have not achieved the primary and secondary outcomes during the study period. If an outcome is missing for $<15 \%$ of participants, analyses will use a complete-case approach. If an outcome is missing for $\geq 15 \%$ of participants, analyses will use multiple imputation.

\section{Secondary analyses}

The intervention and control arms will also be compared for differences in proportions of men achieving secondary outcomes, including confirmed HBsAg test uptake (independent of anti-HCV IgG test uptake), confirmed anti-HCV IgG test uptake (independent of HBsAg test uptake), self-reported HBsAg and anti-HCV IgG test uptake, HBV vaccination, and HIV and STI test uptake during the four-week study period. The analysis approach will be as described for the primary outcome. Change in stigma toward people living with HBV from baseline to week four will be calculated and compared between the intervention and control arms using a Wilcoxon ranksum test. 


\section{Discussion}

This study is unique in terms of setting, population, intervention type, and outcome ascertainment, and will therefore make important contributions to the existing literature. A recent systematic review found that few controlled trials have previously evaluated interventions to increase HBV and HCV test uptake [26]. Only one included study had been conducted in a middle-income country, and none had investigated hepatitis testing among MSM [26]. This RCT will extend our understanding of hepatitis testing interventions by investigating MSM in China, a middle-income country with a high burden of hepatitis.

While crowdsourcing has been used to increase HIV testing and condom use, $[23,24]$ this innovative type of intervention has not been previously applied to HBV and HCV test promotion. By disseminating test promotion materials through social media and encouraging participants to generate content, this crowdsourced intervention has the potential to reach previously underserved MSM and improve hepatitis testing among a population with poor test uptake. Additionally, social media platforms are frequently used by MSM inside and outside China to share content, including health promotion materials [35]. The results of this trial will therefore have implications for future hepatitis testing campaigns targeting MSM in settings with wide-spread social media utilization.

Finally, past studies of hepatitis testing behaviours among MSM have relied on self-report to ascertain whether men had previously received testing [36-38]. However, the reliability of self-reported HBV and HCV test uptake is unknown and potentially subject to recall, ascertainment, and social acceptability biases. This RCT will confirm HBV and HCV test uptake through electronic submission of test report photos. This novel method of outcome ascertainment will provide researchers with reliable biomarker data by verifying testing uptake through hospital or clinic documentation. Participants will be able to receive testing at any facility of their choosing. Because test uptake will not be limited to a specific region or clinical context, the generalizability of results will be enhanced, and this study will be able to inform the design of future hepatitis testing services across a wide-variety of settings.

\section{Additional files}

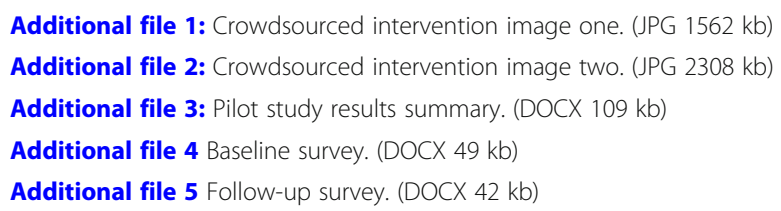

\section{Abbreviations}

HBV: Hepatitis B Virus; HCV: Hepatitis C Virus; HIV: Human Immunodeficiency Virus; LMIC: Low- and Middle-Income Countries; MSM: Men Who Have Sex with Men; RCT: Randomized Controlled Trial; STI: Sexually Transmitted Infection; WHO: The World Health Organization

\section{Acknowledgements}

Authors would like to acknowledge the following persons for their contributions to this research project and manuscript: Tianqi Zhang, Angel Xiaohan Ji, Yang Zhao, Jun Tao, Jason Ong, Tracy Hongyun Fu, and Wei Shufang.

\section{Ethical approval and consent to participate}

The study protocol was approved by IRB bodies at Sun Yat-sen University and University of North Carolina at Chapel Hill prior to launching (IRB Number: 18-0251). Protocol modifications will be submitted to relevant IRB bodies and detailed on Clinicaltrials.gov. All participants will need to complete and agree to an online consent form immediately prior to survey initiation. The form will explain the personal data to be collected as well as how the data will be used for research purposes. Risk and benefits will be explained, and strategies for protecting participant privacy will be described.

\section{Funding}

This work is supported in part by the Doris Duke Charitable Foundation through a grant supporting the Doris Duke International Clinical Research Fellows Program at University of North Carolina at Chapel Hill. TF is a Doris Duke International Research Fellow. This work is also supported by the National Institutes of Health (National Institute of Allergy and Infectious Diseases 1R01Al1 14310-01), UNC CFAR (P30 AI50410), and SESH Global (www.seshglobal.org). This work also receives administrative assistance from the Guangzhou Eighth People's Hospital, UNC Chapel Hill, and UNC - Project China. The listed funders played no role in the development or implementation of this study.

\section{Availability of data and materials}

The results of this study will be prepared and submitted for publication in a peer-reviewed journal. Study findings will also be shared through conference abstracts and presentations, workshops, and to our partnering organizations. All de-identified data generated or analysed during this study will be included in published articles and supplementary information files.

\section{Authors' contributions}

TF and JDT conceived the study. TF, PC, FC, WG, WT, YC, and JDT contributed to intervention development. TF, KZ, WG, KM, YC, and JDT contributed to protocol design. YC and JDT provided oversight. TF and WG wrote the initial draft, and TF oversaw the editing process, with input from $K Z, P C, Y C, K M$, and JDT. All authors read and authorized the final manuscript before submission.

Consent for publication

Not applicable.

\section{Competing interests}

JDT and WT are unpaid advisors at SESH Global LLC. The other authors declare that they have no competing interests.

\section{Publisher's Note}

Springer Nature remains neutral with regard to jurisdictional claims in published maps and institutional affiliations.

\section{Author details}

${ }^{1}$ School of Medicine, University of Washington, Seattle, WA, USA. 2Department of Gastroenterology and Hepatology, University of California San Francisco, San Francisco, USA. ${ }^{3}$ School of Sociology and Anthropology, Sun Yat-sen University, Guangzhou, China. ${ }^{4}$ Division of Communicable Disease, World Health Organization Western Pacific Regional Office, Manila, Philippines. ${ }^{5}$ Department of Laboratorial Science and Technology, Peking University, Beijing, China. ${ }^{6}$ UNC - Project China, University of North Carolina at Chapel Hill, Chapel Hill, NC, USA. ${ }^{7}$ School of Medicine, University of North Carolina at Chapel Hill, Chapel Hill, NC, USA. ${ }^{8}$ Gillings School of Global Public 
Health - Health Policy and Management, University of North Carolina at Chapel Hill, Chapel Hill, NC, USA.

\section{Received: 24 April 2018 Accepted: 20 September 2018 Published online: 29 September 2018}

\section{References}

1. Global Health Estimates. Deaths by cause, age, sex, by country and by region, 2000-2015. Geneva: World Health Organization; 2015. p. 2016.

2. Global Hepatitis Report 2017. Geneva: World Health Organization; 2017.

3. Cui Y, Jia J. Update on epidemiology of hepatitis B and C in China. J Gastroenterol Hepatol. 2013;28(Suppl 1):7-10.

4. Gower E, Estes C, Blach S, Razavi-Shearer K, Razavi H. Global epidemiology and genotype distribution of the hepatitis C virus infection. J Hepatol. 2014; 61:S45-57.

5. Inoue T, Tanaka Y. Hepatitis B virus and its sexually transmitted infection an update. Microbial cell. 2016;3:420-37.

6. Chan DP, Sun HY, Wong HT, Lee SS, Hung CC. Sexually acquired hepatitis C virus infection: a review. Int J Infect Dis. 2016;49:47-58.

7. van Houdt R, Bruisten SM, Speksnijder AG, Prins M: Unexpectedly high proportion of drug users and men having sex with men who develop chronic hepatitis B infection. J Hepatol 2012;57:529-533.

8. Nadol P, O'Connor S, Duong H, Mixson-Hayden T, Tram TH, Xia GL, Kaldor J, Law M, Nguyen T. High hepatitis C virus (HCV) prevalence among men who have sex with men (MSM) in Vietnam and associated risk factors: 2010 Vietnam integrated Behavioural and biologic cross-sectional survey. Sex Transm Infect. 2016;92:542-9.

9. Alonso M, Gutzman A, Mazin R, Pinzon CE, Reveiz L, Ghidinelli M. Hepatitis C in key populations in Latin America and the Caribbean: systematic review and meta-analysis. Int J Public Health. 2015;60:789-98.

10. Pitasi MA, Bingham TA, Sey EK, Smith AJ, Teshale EH. Hepatitis B virus (HBV) infection, immunity and susceptibility among men who have sex with men (MSM), Los Angeles County, USA. AIDS Behav. 2014;18(Suppl 3):248-55.

11. Chow EP, Tucker JD, Wong FY, Nehl EJ, Wang Y, Zhuang X, Zhang L. Disparities and risks of sexually transmissible infections among men who have sex with men in China: a meta-analysis and data synthesis. PLoS One. 2014;9:e89959.

12. Wang C, Wang Y, Huang X, Li X, Zhang T, Song M, Wu L, Du J, Lu X, Shao S, et al. Prevalence and factors associated with hepatitis B immunization and infection among men who have sex with men in Beijing, China. PloS One. 2012;7:e48219.

13. Pan SW, Stein G, Bayus B, Tang W, Mathews A, Wang C, Wei C, Tucker JD: Systematic review of innovation design contests for health: spurring innovation and mass engagement. BMJ Innov. 2017;3:227-37

14. Coffin PO, Scott JD, Golden MR, Sullivan SD. Cost-effectiveness and population outcomes of general population screening for hepatitis C. Clin Infect Dis. 2012:54:1259-71.

15. Geue C, Wu O, Xin Y, Heggie R, Hutchinson S, Martin NK, Fenwick E, Goldberg D, Consortium, ECDC. Cost-Effectiveness of HBV and HCV Screening Strategies--A Systematic Review of Existing Modelling Techniques. PloS One. 2015;10:e0145022.

16. Chonwattana W, Raengsakulrach B, Holtz TH, Wasinrapee P, Tongtoyai J, Chaikummao S, Pattanasin S, McNicholl JM, van Griensven F, Curlin ME. Hepatitis B vaccination uptake and correlates of serologic response among HIV-infected and uninfected men who have sex with men (MSM) in Bangkok, Thailand. Vaccine. 2016;34:2044-50

17. Fitzpatrick T, Pan SW, Tang W, et al HBV and HCV test uptake and correlates among men who have sex with men in China: a nationwide cross-sectional online survey Sex Transm Infect Published Online First: 19 May 2018. https:// doi.org/10.1136/sextrans-2018-053549.

18. Tucker JD, Tang W, Li H, Liu C, Fu R, Tang S, Cao B, Wei C, Tangthanasup TM: Crowdsourcing designathon: a new model for multisectoral collaboration. BMJ Innovations. 2018;4:46-50.

19. Brabham DC, Ribisl KM, Kirchner TR, Bernhardt JM. Crowdsourcing applications for public health. Am J Prev Med. 2014;46:179-87.

20. Zhang Y, Kim JA, Liu F, Tso LS, Tang W, Wei C, Bayus BL, Tucker JD. Creative contributory contests to spur innovation in sexual health: 2 cases and a guide for implementation. Sex Transm Dis. 2015;42:625-8.

21. Parvanta C, Roth Y, Keller H. Crowdsourcing 101: a few basics to make you the leader of the pack. Health Promot Pract. 2013;14:163-7.
22. Prill RJ, Saez-Rodriguez J, Alexopoulos LG, Sorger PK, Stolovitzky G. Crowdsourcing network inference: the DREAM predictive signaling network challenge. Sci Signal. 2011;4:mr7.

23. Liu C, Mao J, Wong T, Tang W, Tso LS, Tang S, Zhang Y, Zhang W, Qin Y, Chen $Z$, et al. Comparing the effectiveness of a crowdsourced video and a social marketing video in promoting condom use among Chinese men who have sex with men: a study protocol. BMJ Open. 2016;6:e010755.

24. Tang W, Han L, Best J, Zhang Y, Mollan K, Kim J, Liu F, Hudgens M, Bayus B, Terris-Prestholt F, et al. Crowdsourcing HIV test promotion videos: a noninferiority randomized controlled trial in China. Clin Infect Dis. 2016;62: 1436-42.

25. Cao B, Liu C, Durvasula M, Tang W, Pan S, Saffer AJ, Wei C, Tucker JD. Social media engagement and HIV testing among men who have sex with men in China: a Nationwide cross-sectional survey. J Med Internet Res. 2017;19: e251.

26. Zhou K, Fitzpatrick T, Walsh N, Kim JY, Chou R, Lackey M, Scott J, Lo YR, Tucker JD. Interventions to optimise the care continuum for chronic viral hepatitis: a systematic review and meta-analyses. Lancet Infect Dis. 2016;16: 1409-22.

27. Spring 2016 Global attitudes survey. Pew Research Center 2016. http:// www.pewglobal.org/dataset/spring-2016-survey-data. Accessed 20 Feb 2018.

28. Statistical Report on Internet Development in China (January 2017). In: statistical report on internet development in China. Vol. 39. Internet network information center. https://cnnic.com.cn/IDR/ReportDownloads/201706/ P020170608523740585924.pdf. Accessed 20 Feb 2018.

29. 2017 WeChat Data Report. In: WeChat Lifestyle Update for Tencent Global Partners Conference. 2017. http://blog.wechat.com/2017/11/09/the-2017wechat-data-report. Accessed 20 Feb 2018.

30. Newsum AM, Stolte IG, van der Meer JT, Schinkel J, van der Valk M, Vanhommerig JW, Buve A, Danta M, Hogewoning A, Prins M et al: Development and validation of the HCV-MOSAIC risk score to assist testing for acute hepatitis $\mathrm{C}$ virus (HCV) infection in HIV-infected men who have sex with men (MSM). Euro Surveill 2017;22:30540.

31. Group SS, Tucker JD. Crowdsourcing to promote HIV testing among MSM in China: study protocol for a stepped wedge randomized controlled trial. Trials. 2017:18:447

32. Watson J, Tang W, Pan S, Wu D, Zhao P, Cao B, Liu C, Bien C, Huang W, Luo Z, et al. Out of the closet, into the clinic: opportunities for expanding MSMcompetent Services in China. Sex Transm Dis. 2018. https://doi.org/10.1097/ OLQ.0000000000000808.

33. Eaton LA, Driffin DD, Kegler C, Smith H, Conway-Washington C, White D, Cherry $C$. The role of stigma and medical mistrust in the routine health care engagement of black men who have sex with men. Am J Public Health. 2015;105:e75-82.

34. McKirnan DJ, Du Bois SN, Alvy LM, Jones K. Health care access and health behaviors among men who have sex with men: the cost of health disparities. Health Educ Behav. 2013:40:32-41.

35. Tso LS, Tang W, Li H, Yan HY, Tucker JD. Social media interventions to prevent HIV: a review of interventions and methodological considerations. Curr Opin Psychol. 2016;9:6-10

36. Rhodes SD, Diclemente RJ, Yee LJ, Hergenrather KC. Factors associated with testing for hepatitis $C$ in an internet-recruited sample of men who have sex with men. Sex Transm Dis. 2001;28:515-20.

37. Brener L, Ellard J, Murphy D, Callander D. Perceptions and deflections: associations between attitudes towards people with hepatitis $C$ and testing for hepatitis C among Australian gay and bisexual men. Sex Health. 2013;10: 268-74

38. Toleran DE, Friese B, Battle RS, Gardiner P, Tran PD, Lam J, Cabangun B. Correlates of HIV and HCV risk and testing among Chinese, Filipino, and Vietnamese men who have sex with men and other at-risk men. AIDS Educ Prev. 2013;25:244-54.

39. Li D, Tang T, Patterson M, Ho M, Heathcote J, Shah $\mathrm{H}$. The impact of hepatitis B knowledge and stigma on screening in Canadian Chinese persons. Can J Gastroenterol. 2012;26:597-602. 